Reprint from Proceedings of the First International African Symposium on Exotic Nuclei (IASEN 2013), iThemba LABS, Cape

Town, South Africa, 1.-6.12.2013,

World Scientific, Singapore (2014), 563-573

http://dx.doi.org/10.1142/9789814632041_0061

\title{
Emission Channeling with Short-Lived Isotopes (EC-SLI) at CERN's ISOLDE facility
}

\author{
U. Wahl ${ }^{\dagger}$, J.G. Correia, A. Costa, and E. David-Bosne \\ Centro de Ciências e Tecnologias Nucleares (C2TN), \\ Instituto Superior Técnico, Universidade de Lisboa, \\ 2686-953 Sacavém, Portugal \\ ${ }^{\dagger}$ E-mail: uwahl@ctn.tecnico.ulisboa.pt
}

L. M. C. Pereira, L. M. Amorim, V. Augustyns, K. Temst, and A. Vantomme,

Instituut voor Kern- en Stralingsfysica (IKS), KU Leuven,

3001 Leuven, Belgium

M. R. da Silva

Centro de Física Nuclear da Universidade de Lisboa (CFNUL), 1649-003 Lisboa, Portugal

D. J. Silva and J. P. Araújo

Departamento de Física, Universidade do Porto, 4169-007 Porto, Portugal

P. Miranda

University of Chile, Santiago, Chile

K. Bharuth-Ram

University of KwaZulu Natal, Durban 4041, South Africa

\begin{abstract}
We give an overview on the historical development and current program for lattice location studies at CERN's ISOLDE facility, where the EC-SLI (Emission Channeling with Short-Lived Isotopes) collaboration maintains several setups for this type of experiments. We illustrate that the three most decisive factors for the success of the technique are access to facilities producing radioactive isotopes, position-sensitive detectors for the emitted decay particles, and reliable simulation codes which allow for quantitative analysis.

Keywords: emission channeling; lattice location; ISOLDE on-line isotope separator
\end{abstract}

\section{Introduction}

Emission channeling (EC) relies on implanting single crystals with radioactive probe atoms that decay by the emission of charged particles such as $\alpha, \beta^{-}$or $\beta^{+}$particles or conversion electrons, which, on their way out of the crystal, experience channeling or blocking effects along crystallographic axes and planes. The resulting anisotropic particle emission yield from the crystal depends in a characteristic way on the lattice sites occupied by the emitter atoms and is recorded with the aid of position sensitive detectors. Quantitative analysis is performed by fitting experimental emission yields in the vicinity of major crystallographic directions by theoretical patterns calculated for emitter atoms on different lattice sites. In comparison to conventional lattice location techniques by means of ion beam channeling, e.g. Rutherford Backscattering/Channeling (RBS/C), the main benefits of emission channeling are 
a roughly four orders of magnitude higher efficiency and the ability to easily study also elements lighter than the host atoms. These facts allow performing detailed lattice location studies with very good statistical accuracy at low fluences of implanted probe atoms, usually as a function of implantation or annealing temperature of the very same sample, which is not feasible by other methods.

Several review papers addressing various aspect of the emission channeling and blocking method have been published in the past [1-8]. In this contribution we approach the subject by giving a historical overview of the development of the technique. As will be shown, the combination of three key ingredients played a crucial role in establishing emission channeling as a technique that nowadays accounts for a significant part of lattice location studies found in the literature: a) facilities where beams of radioactive ions can be produced and implanted into materials, b) position-sensitive detectors suitable for the specific particles emitted in nuclear decay, c) simulation codes that allow to reliably predict the angular-dependent emission patterns for different lattice sites. Some selected examples will be used to illustrate recent fields of work of on-line emission channeling experiments at CERN's ISOLDE facility, including a brief outlook on future developments.

\section{Historical remarks}

Table 1 indicates the milestones in the development of the emission channeling and blocking technique for lattice location studies. Approximately at the same time mid of the 1960s as first experiments were demonstrating the channeling effect of charged particles from an ion beam, experiments were performed also with radioactive isotopes embedded in single crystals. Following the implantation of the $\alpha$ emitter ${ }^{222} \mathrm{Rn}\left(t_{1 / 2}=3.82 \mathrm{~d}\right)$ into a $\mathrm{W}$ single crystal and by measuring the angular distribution of the yield of $5.5 \mathrm{MeV} \alpha$ particles emerging from the crystal, Domeij and Björkvist first observed axial and planar blocking effects $[9,10]$. The emission channeling effect of conversion electrons from ${ }^{133} \mathrm{Xe}(5.24 \mathrm{~d})$ in Ta was discovered as well by Domeij et al shortly thereafter $[11,12]$, followed by emission channeling of electrons and positrons from ${ }^{64} \mathrm{Cu}(12.7 \mathrm{~h})$ in Cu by Uggerhøj and Andersen et al [12,13] and

${ }^{175} \mathrm{Yb}(4.19 \mathrm{~d})$ in $\mathrm{Si}$ [13]. While in all these cases the radioactive isotopes were introduced into the samples by means of an ion implanter at the home laboratories, their origin or preparation was not described in any of the publications.

Table 1. Milestones of the emission channeling technique

\begin{tabular}{|c|c|c|}
\hline Year & Milestone & Refs. \\
\hline 1965 & $\begin{array}{l}\text { First } \alpha \text { blocking lattice location experiment by Domeij and } \\
\text { Bjørkvist (Stockholm) }\end{array}$ & {$[9,10]$} \\
\hline 1965 & $\begin{array}{l}\text { Emission channeling with conversion electrons by Astner, Domeij } \\
\text { et al (Stockholm) }\end{array}$ & {$[10,11]$} \\
\hline 1965 & $\begin{array}{l}\text { Emission channeling with } \beta^{-} \text {and } \beta^{+} \text {by Uggerh } ø j \text { and Andersen } \\
\text { (Aarhus) }\end{array}$ & {$[12,13]$} \\
\hline 1981 & $\begin{array}{l}\text { Revival of emission channeling by Lindner, Wichert, Deicher, and } \\
\text { Recknagel (Konstanz) }\end{array}$ & {$[1,14]$} \\
\hline 1984 & $\begin{array}{l}\text { First use of "manybeam" simulation code for electron emission } \\
\text { channeling (Hofsäss, Lindner et al) }\end{array}$ & $\begin{array}{l}{[1,4,5,7,} \\
15]\end{array}$ \\
\hline 1985 & $\begin{array}{l}\text { First emission channeling experiments at ISOLDE/CERN by } \\
\text { Konstanz group (Hofsäss, Lindner et al) }\end{array}$ & {$[1-5,16]$} \\
\hline 1990 & $\begin{array}{l}\text { Introduction of position-sensitive detection (PSD) for } \alpha \text { emission } \\
\text { channeling, using resistive charge division PSDs (Wahl, Hofsäss et } \\
\text { al) }\end{array}$ & $t^{[2,5,6,17]}$ \\
\hline 1991 & $\begin{array}{l}\text { FLUX ion beam channeling code adapted for simulations of } \alpha \\
\text { emission channeling (Wahl, Hofsäss et al) }\end{array}$ & {$[5,6,18]$} \\
\hline 1996 & $\begin{array}{l}\text { First use of Si pad PSDs ( } 22 \times 22 \text { pixels, } 25 \text { events/s) for off-line } \\
\text { electron emission channeling (Wahl, Correia, Jahn, Vantomme, et } \\
\text { al from IKS Leuven, ITN Sacavém, and ISOLDE) }\end{array}$ & {$[7,19,20]$} \\
\hline 2007 & $\begin{array}{l}\text { ISOLDE on-line electron emission channeling setup equipped with } \\
\text { fast Si pad PSDs ( } 3000 \text { events/s) for short-lived isotopes (EC-SLI } \\
\text { collaboration) }\end{array}$ & {$[21]$} \\
\hline
\end{tabular}




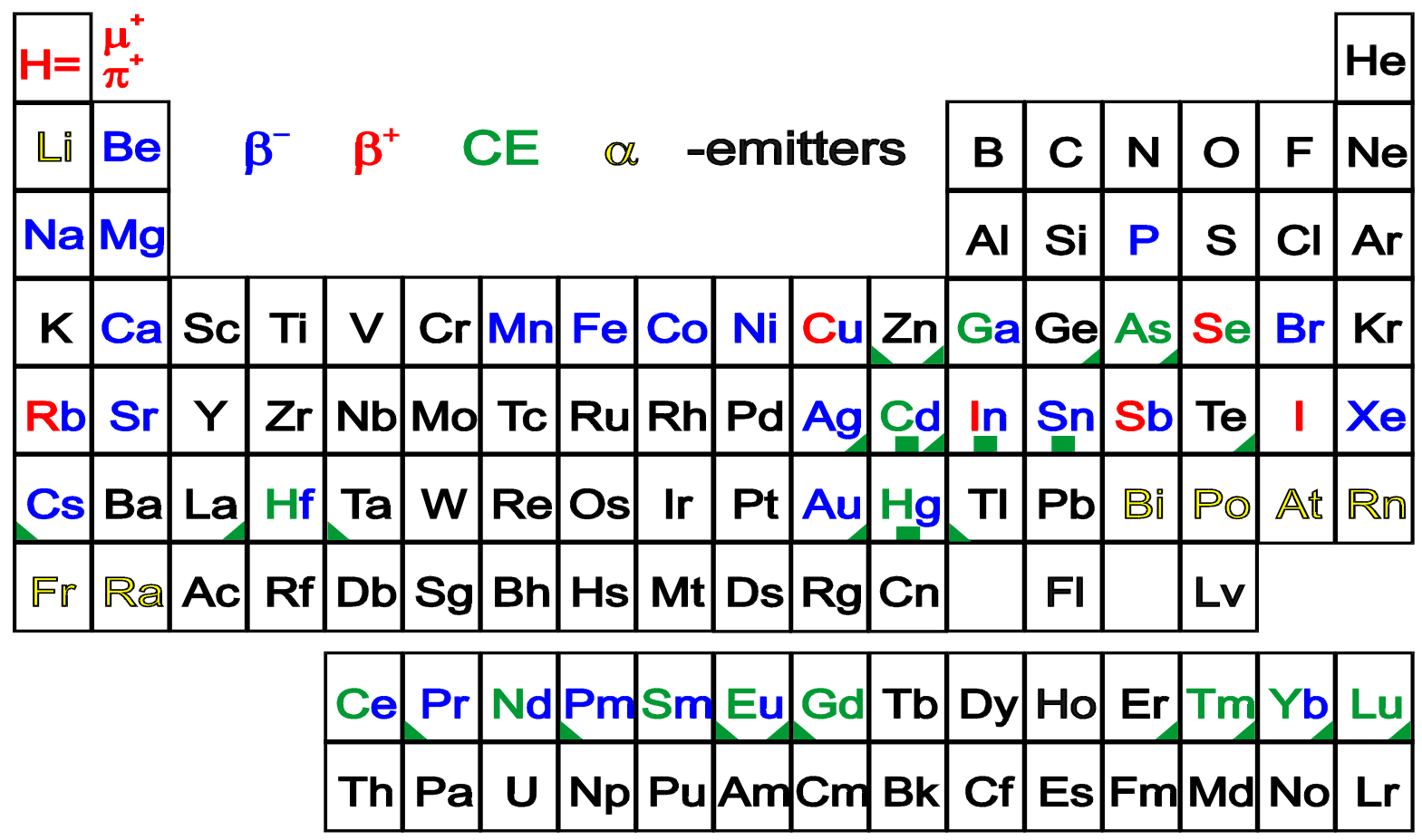

Fig. 1. To date, emission channeling experiments have been successfully performed for radioactive isotopes of the elements marked in the periodic table shown. The different letter styles identify the type of charged particles used: blue symbolizes $\beta^{-}$, red $\beta^{+}$, yellow with black outlined $\alpha$. Elements shown in green letters symbolize parent isotopes of decays that lead to neighbouring daughter nuclei that emit conversion electrons (CEs) and are indicated by the green triangles. Green squares indicate CEs emitted from long-lived isomeric states. Hydrogen has been included in the list due to the chemical similarity of protons with radioactive muons and pions.

Although it was realized at that time that emission channeling and blocking was capable of giving direct information on the lattice sites of the radioactive probe atoms, it was not established immediately as a major experimental method for that purpose. This was due to the fact that the number of suitable and easily available radioisotopes, as well as the access to ion implanters that could be used with radioactive probes, was limited. However, in the 1980s the technique was revived by Lindner, Wichert, Deicher and Recknagel from the University of Konstanz in Germany. Their work was motivated by the wish to combine studies characterizing the atomic surroundings of a radioactive nucleus by means of hyperfine interaction methods such as perturbed angular correlation (PAC) or Mössbauer Spectroscopy (MS) directly with emission channeling lattice location experiments performed on the same sample. The first experiments in 1981 were carried out using the well-known PAC probe atom ${ }^{111} \mathrm{In}\left(t_{1 / 2}=2.80 \mathrm{~d}\right)$, which was implanted in single crystals of $\mathrm{Cu}[1,14]$. The channeling effects of $145 \mathrm{keV}$ and $219 \mathrm{keV} \mathrm{K}$-conversion electrons proved that the ${ }^{111} \mathrm{In}$ occupied substitutional lattice sites in $\mathrm{Cu}$. The ${ }^{111} \mathrm{In}$ isotope used for the studies was commercially available and could therefore be bought and introduced into the samples at the home laboratory by means of an ion implanter. However, in order to make the method more versatile, access to a wider range of radioactive isotopes was needed, and in 1985 the group therefore began to conduct experiments at the ISOLDE on-line isotope separator facility at CERN [1-5,16]. At ISOLDE, currently more than 800 different radioisotopes of 60-70 chemical elements can be produced by nuclear reactions and are available as $30-60 \mathrm{keV}$ ion beams, which is a very suitable energy range for implantation of emission channeling probes. 


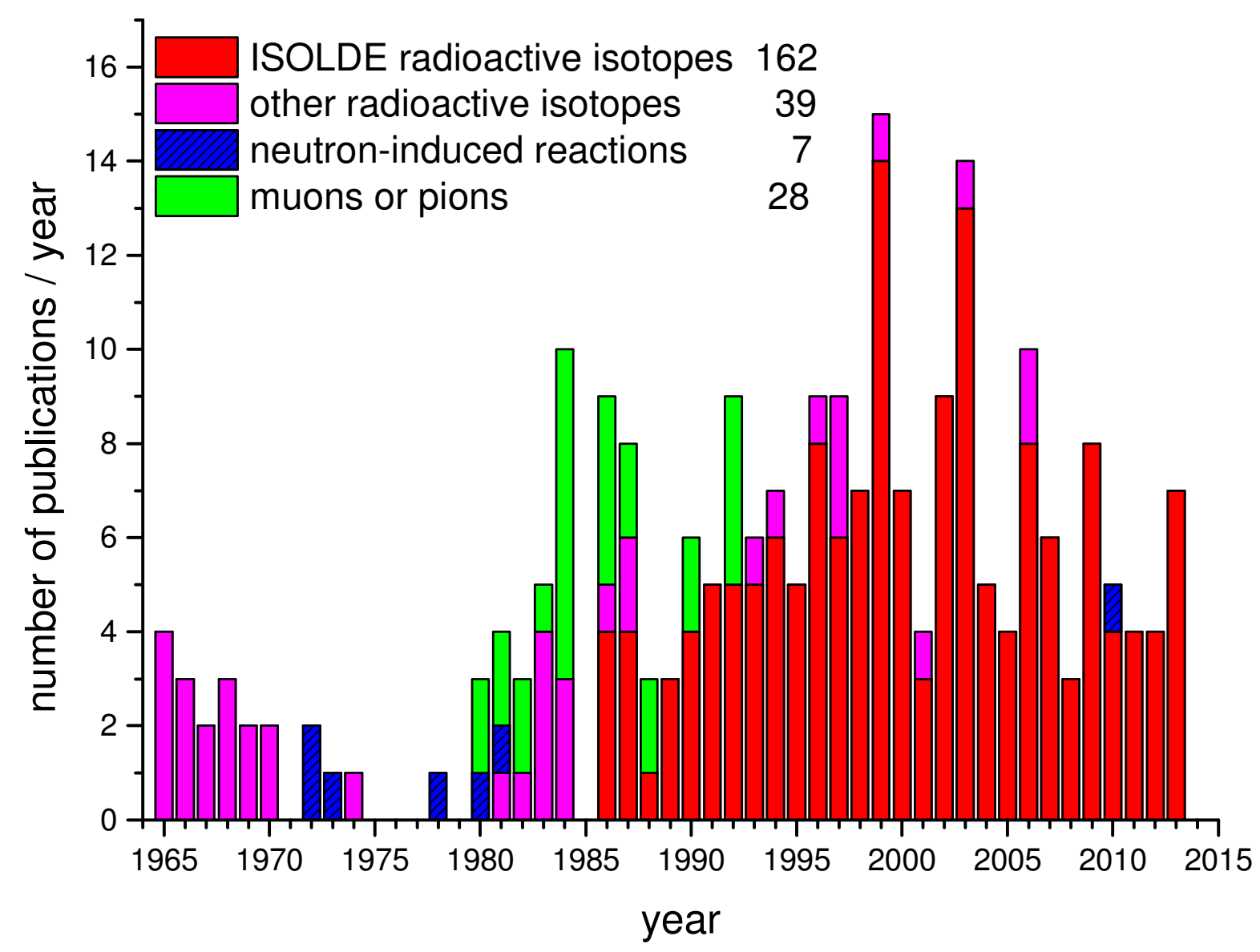

Fig. 2. Publication history of emission channeling experiments for lattice location purposes, based on 236 publications and theses on the subject known to the authors.

In 1984 the quantum-mechanical "manybeam" formalism had been successfully adapted in order to simulate electron emission channeling effects $[1,15]$. However, it took about 10 more years until this approach had been developed into a truly quantitative method of analysis [4,5], and considerable progress in computing power was required before it could become routinely used [7].

The year 1990 saw the first application of position-sensitive detectors (PSDs) using the principle of resistive charge division for the detection of $\alpha$ emission channeling [2,5,6,17]. The method of obtaining two-dimensional patterns recorded by a PSD instead of rotating the sample step by step in front of a collimated non-position-sensitive detector (which was used in all previous experiments) increased the efficiency of the experiments by about 1-2 orders of magnitude. This experimental breakthrough was closely followed by adapting the Monte Carlo ion beam simulation code FLUX for applications in $\alpha$ emission channeling $[5,6,18]$, which then also allowed fully quantitative analysis of this type of experiments.

In 1996 a further milestone was the implementation of Si pad detectors, consisting of $22 \times 22$ pixels ( $1.4 \mathrm{~mm}$ pixel size), for the detection of electron emission channeling $[7,19,20]$. While the first such PSD was only capable of taking data at a count rate of $\sim 25$ events/s, it proofed already an excellent tool for lattice location experiments of long-lived radioisotopes emitting $\beta$ particles or conversion electrons. When new readout systems allowed to upgrade the count rate of the pad PSDs to $\sim 3000$ events/s, the time was ripe to finally install an on-line setup equipped with such a PSD for electron emission channeling with short-lived isotopes at one of the beam lines at ISOLDE in 2007 [21]. The EC-SLI collaboration (Emission Channeling with Short-Lived Isotopes) currently consists of 14 scientists from 6 home institutes, mostly in Portugal and Belgium, but also South Africa and Chile. Besides the on-line setup 
for short-lived isotopes, three more experimental setups are available for off-line experiments with long-lived isotopes at ISOLDE.

To date, around 238 publications and theses are known to the authors that address emission channeling and blocking lattice location experiments, reporting the use of $\sim 80$ different isotopes and decays of $\sim 50$ chemical elements (Figs. 1+2). Besides some work on metals, the vast majority of studies concerned the field of dopants and impurities in semiconductors. Some of the highlights over the years were for instance direct evidence for substitutional ion-implanted indium dopants in Si [22], site changes of ion-implanted $\mathrm{Li}$ in GaAs [23], direct evidence for tetrahedral interstitial $\mathrm{Er}$ in $\mathrm{Si}$ [24], lattice location and stability of ion implanted $\mathrm{Cu}$ in $\mathrm{Si}$ [25], direct evidence for As as a $\mathrm{Zn}$-site impurity in $\mathrm{ZnO}$ [26], transition metal impurities on the bond-centered site in Ge [27], and lattice location of rare earth impurities in III-nitrides [28].

\section{Recent examples}

Current emission channeling experiments at ISOLDE focus on two major topics. On the one hand this is the lattice location of transition-metal probes ${ }^{56} \mathrm{Mn}(2.56 \mathrm{~h}),{ }^{59} \mathrm{Fe}(44.5 \mathrm{~d}),{ }^{61} \mathrm{Co}(1.65 \mathrm{~h})$, and ${ }^{65} \mathrm{Ni}(2.52 \mathrm{~h})$ in semiconductors and oxides, where these elements are either unwanted contaminants, e.g. in $\mathrm{Si}, \mathrm{Ge}$ or $\mathrm{SiC}$, or are of interest as magnetic dopants for the realization of dilute magnetic semiconductors, e.g. in $\mathrm{GaN}, \mathrm{ZnO}, \mathrm{GaAs}, \mathrm{SrTiO}_{3}$. The second field addresses the p-type doping of III-nitride semiconductors such as GaN, AlN and InN, which is investigated using the short-lived alkaline earth probes ${ }^{11} \mathrm{Be}(13.8 \mathrm{~min})$ and ${ }^{27} \mathrm{Mg}$ (9.45 min) or the long-lived probes ${ }^{45} \mathrm{Ca}(163 \mathrm{~d})$ and ${ }^{89} \mathrm{Sr}(50.5 \mathrm{~d})$.

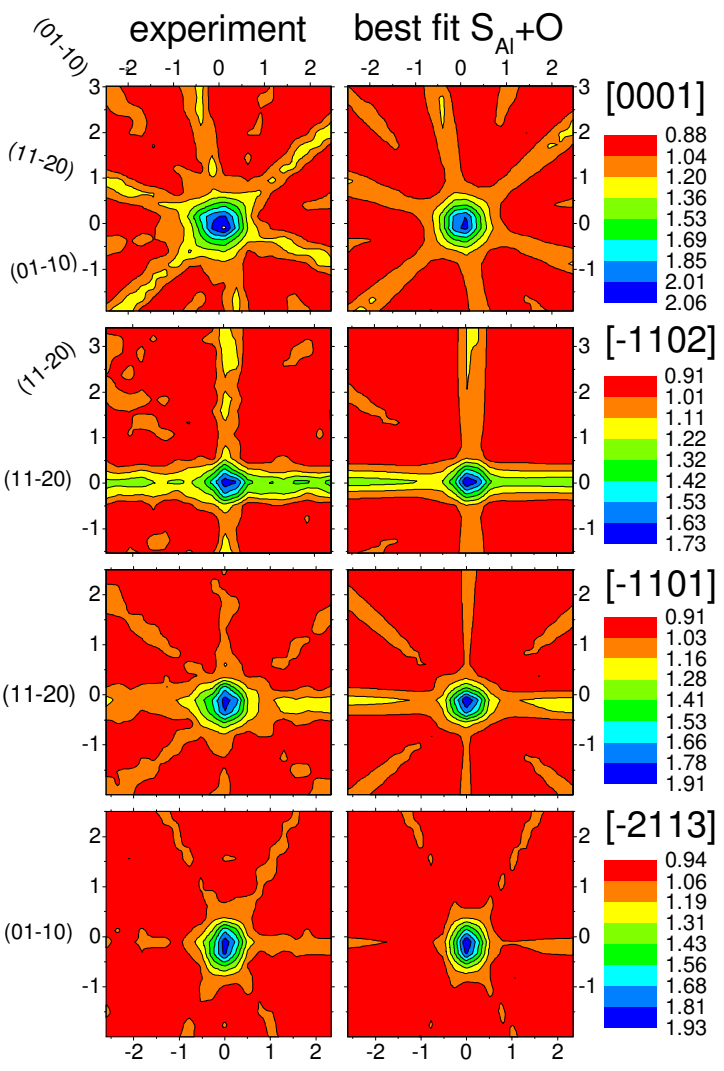

(a)

(b)

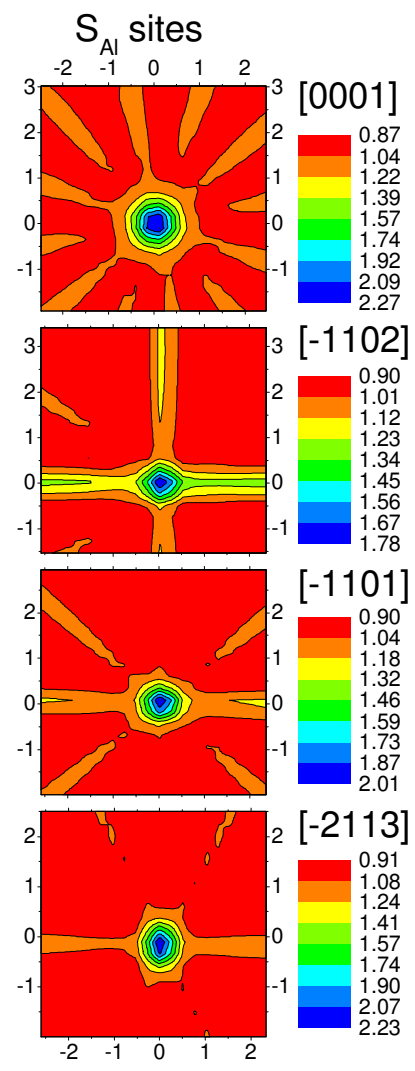

(c)

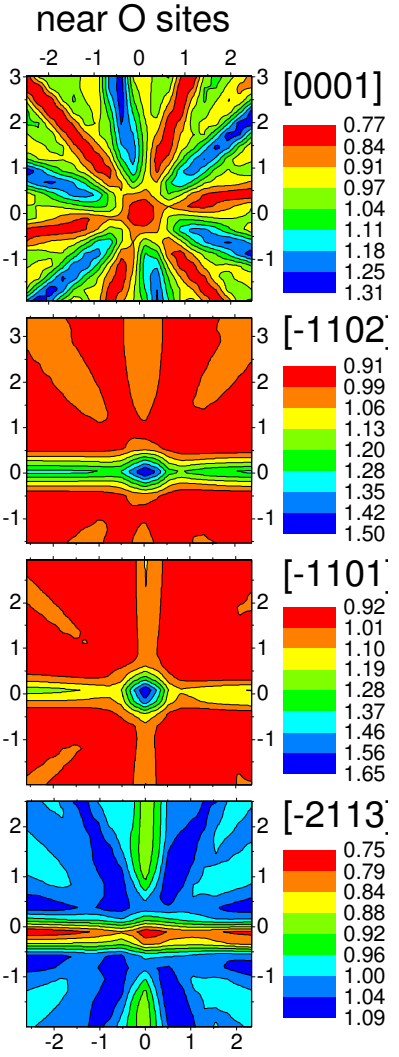

(d)

Fig. 3. Comparison of the experimental normalized angular electron emission yield (a) around [0001], [-1102], [-1101] and [-2113] directions for RT implantation with the best fit of simulated patterns (b). The theoretical patterns for $100 \%$ of ${ }^{27} \mathrm{Mg}$ on substitutional Al sites $\left(\mathrm{S}_{\mathrm{Al}}\right)$ and near octahedral interstitial sites $(\mathrm{O})$ are shown in columns (c) and (d), respectively. 
As an example, Fig. 3 (a) shows the experimental $\beta^{-}$emission channeling patterns around the [0001], [-1102], [-1101] and [-2113] axes of a AlN single-crystalline thin film measured during room temperature implantation of

${ }^{27} \mathrm{Mg}$ [29]. The best fits to the experimental patterns are shown in Fig. 3 (b) and represent a linear combination of $63 \%$ of ${ }^{27} \mathrm{Mg}$ on substitutional $\mathrm{Al}$ sites $\left(\mathrm{S}_{\mathrm{Al}}\right)$ plus $32 \%$ near interstitial octahedral sites $(\mathrm{O})$. For comparison, the theoretical patterns corresponding to $100 \%$ of emitters on $\mathrm{S}_{\mathrm{Al}}$ sites and near $\mathrm{O}$ sites are shown in Fig. 3 (c) and (d), respectively. That the interstitial $\mathrm{O}$ sites contribute to the experimental patterns is clear from the fact that the channeling effects along the (01-10) planes are less intense than is expected for pure substitutional Al site occupancy. A detailed analysis showed that the possible displacements of the substitutional $\mathrm{Mg}$ from ideal $\mathrm{Al}$ sites are at maximum $0.1 \AA$. Performing the measurement for an implantation temperature of $500^{\circ} \mathrm{C}$, the interstitial ${ }^{27} \mathrm{Mg}$ is completely converted to substitutional $\mathrm{Al}$ sites, from which an activation energy of 1.1-1.7 eV can be estimated for the interstitial migration of $\mathrm{Mg}$ in AlN. These experiments represent the first successful lattice location of $\mathrm{Mg}$ in a semiconductor.

\section{Conclusions and outlook}

An overview was given on the historical development of the emission channeling and blocking technique for lattice location of radioactive probes in single crystals. We illustrated that the three most decisive factors for the success of the technique are access to facilities producing radioactive isotopes in sufficient quantity and purity, positionsensitive detectors for the emitted decay particles, and reliable simulation codes which allow for quantitative analysis.

With respect to future milestones, the focus is today on the use of position-sensitive micro-pixel detectors of the Timepix type [30]. With up to $512 \times 512$ pixels of $55 \mu \mathrm{m}$ pixel size this type of PSD provides more than one order of magnitude better position resolution than the currently used pad detectors, allow also for a considerable improvement in angular resolution. It is expected that this will significantly improve the accuracy of the lattice location experiments.

The development of new beams of radioactive isotopes by the ISOLDE target and ion source group is on-going and in particular targets refractory chemical elements which are notoriously difficult to provide as radioactive ion beams. One example is the positron emitting isotope ${ }^{11} \mathrm{C}(20.3 \mathrm{~min})$, which has recently been made available with high yields in form of the molecular ion beam ${ }^{11} \mathrm{C}^{16} \mathrm{O}^{+}$; another is ${ }^{8} \mathrm{~B}(762 \mathrm{~ms})$ where it is currently being explored to provide beams by means of forming and ionizing the di-fluoride molecule ${ }^{8} \mathrm{~B}^{19} \mathrm{~F}_{2}$. This kind of technical development work will continue opening new possibilities for emission channeling experiments with probe atoms for chemical elements that were previously unavailable.

\section{Acknowledgments}

This work was supported by the Portuguese Foundation for Science and Technology (FCT project CERN/FP/123585/2011), FWO Vlaanderen, the KU Leuven (GOA/09/006 and GOA/14/007) and by the European Commission through SPIRIT (Support of Public and Industrial Research using Ion beam Technology, Contract 227012) and ENSAR (European Nuclear Science and Applications Research, Contract 262010). We thank the ISOLDE facility at CERN for providing radioactive beams.

\section{References}

1. H. Hofsäss and G. Lindner: Emission channeling and blocking, Physics Reports 201, 121 (1991).

2. H. Hofsäss, S. Winter, S.G. Jahn, U. Wahl and E. Recknagel, Emission Channeling Studies in Semiconductors, Nucl. Instr. Meth. Phys. Res. B 63, 83 (1992).

3. S. Winter, H. Hofsäss, S.G. Jahn, G. Lindner, U. Wahl and E. Recknagel, Emission channeling, in Hyperfine Interaction of Defects in Semiconductors, ed. G. Langouche (Elsevier, Amsterdam, 1992), pp 157-185.

4. H. Hofsäss, U. Wahl and S.G. Jahn, Impurity lattice location and recovery of structural defects in semiconductors studied by emission channeling, Hyperfine Interactions 84, 27 (1994).

5. H. Hofsäss, Emission channeling, Hyperfine Interactions 97, 247 (1996). 
6. U. Wahl and the ISOLDE collaboration, Emission channeling studies of Li in semiconductors, Physics Reports 280, 145 (1997).

7. U. Wahl, Advances in electron emission channeling measurements in semiconductors, Hyperfine Interactions 129, 349 (2000).

8. U. Wahl, J.G. Correia, E. Rita, E. Alves, J.C. Soares, B. De Vries, V. Matias, A. Vantomme, and the ISOLDE collaboration, Recent emission channeling studies in wide band gap semiconductors, Hyperfine Interactions 158, 363 (2005).

9. B. Domeij and K. Bjørkvist, Anisotropic emission of $\alpha$-particles from a monocrystalline source, Phys. Lett. 14, 127 (1965).

10. B. Domeij and K. Bjørkvist, Crystal lattice effects in the emission of charged particles from monocrystalline sources, Nucl. Instr. Meth. 38, 207 (1965).

11. G. Astner, I. Bergström, B. Domeij, L. Eriksson and Å. Persson, Angular dependence of conversion electrons from a monocrystalline source, Phys. Lett. 14, 308 (1965).

12. E. Uggerhøj, Orientation dependence of the emission of positrons and electrons from ${ }^{64} \mathrm{Cu}$ embedded in single crystals, Phys. Lett. 22, 382 (1966).

13. E. Uggerhøj and J.U. Andersen: "Influence of lattice structure on motion of positrons and electrons through single crystals", Can. J. Phys. 46, 543 (1968).

14. T. Wichert, G. Lindner, M. Deicher and E. Recknagel, Realization of simultaneous channeling and nuclearhyperfine-interaction experiments, Phys. Rev. B 24, 7467 (1981). 